Reprod. Nutr. Dévelop., 1984, 24 (4), 387-403.

\title{
Correction par voie nutritionnelle d'un excès d'acides gras insaturés dans les lipides corporels du rat en croissance
}

\author{
P. GUESNET, Y. DEMARNE, Andrée PIHET
}

Station de Recherches de Nutrition, I.N.R.A., 78350 Jouy-en-Josas, France.

Summary. Correction by nutritional route of an excess of unsaturated fatty acids in body lipids of growing rat.

The use of hydrogenated coconut oil as a putative substance for correcting an excess of unsaturated fatty acids in reserve lipids was tested in growing rats. Young rats with a live weight of about $70 \mathrm{~g}$ were given diet $A$ including $4 \%$ of sunflower oil which very rapidly created a triglyceride store containing 25 to $30 \%$ of linoleic acid (18:2 n-6). At different weights $(200,250,300$ and $350 \mathrm{~g})$, diet A was replaced by diet B containing $15 \%$ of hydrogenated coconut oil so that there was the same number of animals in each group. All the rats were killed at $400 \mathrm{~g}$, and diet-A and B were compared as to final fatty acid composition of body lipids (i.e. values taken all during growth from 70 to $400 \mathrm{~g}$ ).

Hydrogenated coconut oil was a very effective substance for rapidly decreasing levels of reserve lipid linoleic acid and for increasing triglyceride melting-point. The impact on the decrease in total unsaturated fatty acid concentrations was more marked the earlier the diet was replaced. However, the latest replacements (at 300 and $350 \mathrm{~g}$ ) still permitted 70 and $50 \%$, respectively, of the maximal effect observed in rats eating diet $\mathrm{B}$ from $70 \mathrm{~g}$.

The present experiment shows that when coconut oil was introduced in the diet over a period corresponding to the last one-eight of total body growth in rat, the final concentrations of unsaturated fatty acids could still be considerably reduced, particularly that of linoleic acid. In the same way, there was increasing esterification of myristic acid $(14: 0)$, and especially of lauric acid (12:0), which appeared preferentially in sn-1 and sn-3 positions in triglycerides. This esterification of medium-chain fatty acids led to a modification in the mode of other fatty acid distribution. Analysis of liver phospholipids showed that when diet $A$ was replaced above $200 \mathrm{~g}$ by diet $B$, there was no biochemical evidence of any deficiency of essential fatty acids of the $n-6$ series.

\section{Introduction.}

Lorsqu'il reçoit un régime lipidoprive, le mammifère monogastrique en croissance synthétise des triglycérides de réserve riches en acide oléique (18:1 n-9). Chez le Porc ou chez le Rat, les concentrations généralement rapportées pour ces conditions nutritionnelles sont de l'ordre de 40 à $60 \%$ des acides gras totaux (Flanzy, François et Rérat, 1970; Demarne et al., 1981). L'acide oléique est 
préférentiellement estérifié sur les positions sn-1 et sn-3 dans les triglycérides de l'adipocyte de Porc, alors qu'il est estérifié "au hasard", ou d'une façon légèrement préféréntielle sur la position sn-2, dans les triglycérides de l'adipocyte de Rat (Brockerhoff, Hoyle et Wolmark, 1966 ; Christie et Moore, 1970 ; Demarne et al., 1981).

Les tissus adipeux des mammifères monogastriques possèdent une remarquable aptitude à estérifier l'acide linoléique (18:2 n-6) dont l'origine est exclusivement alimentaire. Ainsi, chez le Rat recevant un régime contenant $10 \%$ d'huile de tournesol, $50 \%$ de l'acide linoléique ingéré entre 70 et $300 \mathrm{~g}$ de poids vif est estérifié dans les triglycérides de réserve (Demarne et al., 1975). Chez le Porc en croissance, le coefficient de stockage de l'acide linoléique est supérieur à $50 \%$ et augmente avec la vitesse de croissance (Flanzy, François et Rérat, 1970). L'acide linoléique est estérifié d'une façon très préférentielle sur la position sn-2 chez le Rat, mais au fur et à mesure que sa concentration augmente, il colonise rapidement les positions sn-1 et sn-3 (Demarne et al., 1981). Dans les triglycérides de réserve de Porc, l'acide linoléique occupe très préférentiellement les positions « externes " et plus particulièrement la position sn-3 (Christie et Moore, 1970).

Par rapport à ce qui est observé en régime lipidoprive, un apport de lipides riches en acide linoléique, comme c'est le cas pour l'huile de maïs ou celle de tournesol, diminue sensiblement les teneurs en acide oléique des triglycérides de réserve. Cependant, compte tenu de l'estérification concomitante d'une quantité importante d'acide linoléique, la somme des concentrations en acides gras insaturés en C18 (18:1 + 18:2) augmente très nettement (Flanzy, François et Rérat, 1970 ; Demarne et al., 1981). Ainsi, la somme des acides gras totaux insaturés estérifiés dans les triglycérides de réserve du Rat de $300 \mathrm{~g}$ ayant reçu depuis le sevrage un régime lipidoprive ou un aliment contenant $10 \%$ d'huile de tournesol est respectivement de $45 \%$ et $70 \%$ (Demarne et al., 1981). En même temps, les teneurs en acide palmitique (16:0) diminuent considérablement. Chez le Porc, des altérations du même ordre sont observées (Flanzy, François et Rérat, 1970).

La diminution des concentrations en acides gras saturés à points de fusion élevés $\left(>60^{\circ} \mathrm{C}\right)$, l'augmentation parallèle des concentrations en acides gras insaturés à point de fusion bas $\left(<20^{\circ} \mathrm{C}\right) \mathrm{d}^{\prime} u n e$ part, et les variations du mode de distribution intramoléculaire des acides gras d'autre part, sont susceptibles d'entraîner des modifications sensibles des propriétés physico-chimiques des triglycérides du tissu adipeux. Ainsi, si le point de fusion de la tripalmitine se situe aux environs de $65^{\circ} \mathrm{C}$, il passe à $36^{\circ} \mathrm{C}$ pour la $\beta$ oléo- $\alpha \alpha^{\prime}$ dipalmitine, $28^{\circ} \mathrm{C}$ pour la $\beta$ linoléo- $\alpha \alpha^{\prime}$ - dipalmitine, $18^{\circ} \mathrm{C}$ pour I' $\alpha \beta$ dioléo- $\alpha^{\prime}$-palmitine et $-4{ }^{\circ} \mathrm{C}$ pour l' $\alpha \beta$ dilinoléo- $\alpha^{\prime}$-palmitine (Parisot, 1949), dans la mesure où le mode de cristallisation permet la comparaison.

Au niveau des productions animales et en particulier chez le Porc, l'introduction massive de maïs dans l'alimentation et la sélection de souches à faible adiposité favorisent l'apparition de graisses molles caractérisées par des teneurs élevées en acide linoléique et des points de fusion bas (Sellier, 1983). Au cours de la croissance du Porc et du Rat, l'ingestion de lipides riches en acide laurique (12:0, $\mathrm{PF}=44^{\circ} \mathrm{C}$ ) conduit à l'élaboration d'une graisse de réserve ferme, caractérisée par des triglycérides à basse teneur en acides gras insaturés (Demarne et al., 
1977 ; Demarne et al., 1978). De ce point de vue, l'acide laurique est beaucoup plus efficace que les acides gras saturés à chaîne longue (Demarne et al., 1974). De plus, son coefficient d'utilisation digestive est bien plus élevé (Flanzy, Rérat et François, 1968).

Nous avons donc étudié, par une approche nouvelle, les propriétés nutritionnelles de cet acide gras. Le but du présent travail est de définir l'efficacité de la correction du taux d'acides gras insaturés estérifiés, induite par l'introduction dans la ration et à différents stades de croissance, d'huile de coprah hydrogénée riche en acide laurique. Dans cette expérience de modélisation, notre objectif majeur est de rechercher si des interventions nutritionnelles tardives, et donc plus économiques, sont susceptibles de fournir des résultats nouveaux et intéressants. En plus des effets observés au niveau de la composition en acides gras des triglycérides de réserve, nous étudions également les modifications de la distribution stéréospécifique des acides gras, puisque ce facteur est susceptible d'influencer le point de fusion des graisses de réserve. Enfin, compte tenu du fait que nous employons un régime de correction appauvri en acide linoléique, nous nous attachons à mesurer les éventuels risques d'apparition de carences en acides gras essentiels.

\section{Matériel et méthodes.}

1. Animaux et régimes. - Les expériences sont réalisées en utilisant des rats de souches Wistar (EVIC-CEBA, ZI, F 33290 Blanquefort). Deux régimes expérimentaux sont utilisés (tabl. 1). Le premier, ou régime de base $A$, contient $4 \%$ de lipides introduits sous forme d'huile de tournesol à haute teneur en acide linoléique

\section{TABLEAU 1}

Composition centésimale des régimes

\begin{tabular}{lcc}
\hline \multicolumn{1}{c}{ Composants } & Régime A & Régime B \\
\hline Amidon de maïs & 41,9 & 34,9 \\
Sucre cristallisé & 24,1 & 20,1 \\
Caséine lactique & 22,1 & 22,1 \\
Huile de tournesol $\left({ }^{1}\right)$ & 4,0 & - \\
Huile de coprah hydrogénée $\left({ }^{2}\right)$ & - & 15,0 \\
dL-méthionine & 0,5 & 0,5 \\
Complément minéral $\left({ }^{3}\right)$ & 4,3 & 4,3 \\
Complément vitaminique $\left({ }^{4}\right)$ & - & 3,1 \\
Cellulose colmacel $\mathrm{F}_{1} \mathrm{~F}_{2}$ & 3,1 & - \\
\hline
\end{tabular}

(1) Huile de tournesol: $16: 0=6,6 \% ; 18: 0=4,8 \% ; 18: 1=21,8 \% ; 18: 2=66,8 \%$.

(2) Huile de coprah hydrogénée : $8: 0=6,0 \% ; 10: 0=8,5 \% ; 12: 0=52,3 \% ; 14: 0=16,8 \%$; $16: 0=8,1 \% ; 18: 0=8,3 \%$.

(3) Mélange minéral (composition en \%): $\mathrm{CaCO}_{3}=20,714 ; \mathrm{KCl}=20,857 ; \mathrm{IK}=0,009$; $\mathrm{CaHPO}_{4}, 2 \mathrm{H}_{2} \mathrm{O}=32,285 ; \mathrm{NaHPO}_{4}, 2 \mathrm{H}_{2} \mathrm{O}=17,143 ; \mathrm{MgSO}_{4}, 7 \mathrm{H}_{2} \mathrm{O}=6,571 ; \mathrm{CuSO}_{4}$, $5 \mathrm{H}_{2} \mathrm{O}=0,143 ; \mathrm{MnSO}_{4}, \mathrm{H}_{2} \mathrm{O}=0,440 ; \mathrm{ZnCO}_{3}=0,152 ; \mathrm{Fe}_{3}\left(\mathrm{C}_{6} \mathrm{H}_{5} \mathrm{O}_{2}\right)_{2}, 6 \mathrm{H}_{2} \mathrm{O}=1,686$.

( $\left.{ }^{4}\right)$ Mélange vitaminique (g pour $100 \mathrm{~kg}$ d'aliment) : vitamine $\mathrm{A}(500000 \mathrm{UI} / \mathrm{g}): 4,0 ;$ vitamine $\mathrm{D}_{3}$ $(100000 \mathrm{UI} / \mathrm{g})$; vitamine $\mathrm{E}(250 \mathrm{UI} / \mathrm{g}): 160$ : vitamine $\mathrm{K}: 0,200$; chlorhydrate de thiamine $\left(\mathrm{B}_{1}\right): 2,0$; riboflavine $\left(B_{2}\right): 3,0$; chlorhydrate de pyridoxine $\left(B_{6}\right): 10,0$; acide nicotinique : 2,$2 ;$ acide folique : 0,100 ; acide para-aminobenzoïque : 10,0 ; vitamine $B_{12}: 0,5$; inositol : 20,0 ; biotine (à $1 \%$ ): 2,0 ; pantothénate de $\mathrm{Ca}: 10,0$; niacine : 10,0 . 
$(18: 2 \mathrm{n}-6)$, soit environ $65 \%$ des acides gras totaux. Le second, ou régime de correction $\mathrm{B}$, contient $15 \%$ de lipides totaux introduits sous forme d'huile de coprah hydrogénée dont l'acide gras majeur est l'acide laurique (12:0) présent à une concentration voisine de $50 \%$ des acides gras totaux. Les régimes expérimentaux sont fabriqués par la FMAE (INRA-CNRZ, La Minière, F 78280 Guyancourt).

2. Protocole expérimental. - Le travail présenté comporte trois expérimentations entreprises sur des rats en croissance entre 70 et $400 \mathrm{~g}$ de poids vif. L'expérimentation complète nécessite 63 rats.

Expérience 1. - Elle a pour but d'étudier les effets du régime de base A sur l'évolution des caractéristiques des lipides corporels du Rat en croissance. Dans ce travail de modélisation, le régime $A$ est censé mimer les effets d'un régime « commercial » conduisant à la synthèse de lipides de réserve dont les teneurs en acides gras insaturés sont jugées excessives. Dix-huit jeunes ratons d'un poids moyen d'environ $70 \mathrm{~g}$ sont disposés en cages individuelles et reçoivent le régime $A$ ad libitum. Trois animaux supplémentaires, et n'appartenant pas au lot expérimental, sont abattus à jeun en début d'expérience (témoins initiaux). Aux poids respectifs de $150,200,250,300,350$ et $400 \mathrm{~g}$, trois animaux tirés au sort sont abattus. Les rats à jeun sont décapités sous anesthésie générale. Le foie est immédiatement prélevé et congelé. La carcasse est ensuite broyée dans un hachoir à viande de type "Sharfen ». Après lyophilisation, les broyats et les foies sont conservés à $-80^{\circ} \mathrm{C}$ jusqu'à leur analyse ultérieure.

Expérience 2. - Elle a pour but de définir les effets du régime de correction B sur l'évolution des caractéristiques des lipides corporels du Rat en croissance. Le régime ingéré est celui qui est censé favoriser la synthèse des lipides les plus pauvres en acides gras insaturés en C18. L'expérience 2 permet donc d'obtenir les références nécessaires concernant l'effet maximal du régime de correction. Douze rats maintenus en cages individuelles reçoivent le régime $\mathrm{B}$ ad libitum. Les abattages sont réalisés selon les mêmes procédures que lors de l'expérience 1. Ils ont lieu à $150,200,300$ et $400 \mathrm{~g}$.

Expérience 3. - Elle a pour but d'étudier les effets de correction induits par la substitution du régime de correction $\mathrm{B}$ au régime de base $\mathrm{A}$ à différents stades de la croissance. L'expérience est réalisée en utilisant 4 groupes de rats alimentés ad libitum.

- Le groupe (3-1) est composé de 12 rats qui ingèrent, de $70 \mathrm{~g}$ à $200 \mathrm{~g}$, le régime de base $A$. A partir de $200 \mathrm{~g}$, le régime $A$ est remplacé par le régime $B$. Par groupe de 3 , et dans les conditions précédemment décrites, les animaux sont abattus à $250,300,350$ et $400 \mathrm{~g}$ de poids vif.

- Le groupe (3-2) est composé de 9 rats pour lesquels le changement de régime s'opère à $250 \mathrm{~g}$. Les abattages ont lieu à 300,350 et $400 \mathrm{~g}$ de poids vif.

- Le groupe (3-3) est composé de 6 rats pour lesquels le changement de régime s'opère à $300 \mathrm{~g}$ et les abattages à 350 et $400 \mathrm{~g}$.

- Enfin, le groupe (3-4) est composé de 3 rats pour lesquels le changement de régime a lieu à $350 \mathrm{~g}$ et l'abattage à $400 \mathrm{~g}$. 


\section{Méthodes analytiques.}

Extraction des lipides corporels et analyse des acides gras. - L'extraction des lipides est réalisée en utilisant la méthode proposée par Folch, Lees et SloaneStanley (1957). Les lipides totaux ainsi obtenus sont ensuite saponifiés à froid, en employant une solution de potasse alcoolique à $10 \%$. Cette saponification permet de séparer les acides gras des autres constituants lipidiques. Après hydrolyse des savons, les acides gras purifiés provenant des différents échantillons sont transformés en esters méthyliques ou butyliques selon que l'extrait contient ou ne contient pas d'acides gras à chaîne moyenne $(\leqslant C 14)$.

La composition en acides gras des différents extraits est déterminée par chromatographie en phase gazeuse. Les conditions d'analyse sont les suivantes: colonne capillaire en verre de diamètre interne de $0,3 \mathrm{~mm}$ et d'une longueur de $47 \mathrm{~m}$, appareil Girdel 75-FD-2, phase stationnaire composée de FFAP, le gaz vecteur est de l'hydrogène dont la pression, au niveau de l'injecteur, est de 0,5 bar, la température du four est de $196^{\circ} \mathrm{C}$ et la détection est réalisée par ionisation de flamme. L'appareillage est couplé à un calculateur-intégrateur LTT 4212. La nature des différents acides gras séparés sont déterminés par calcul de leur longueur équivalente de chaîne (LEC).

De façon à mesurer l'importance des éventuelles sous-estimations des concentrations en acides gras à chaîne moyenne obtenues par cette méthode, un mélange de triglycérides dont la composition en acides gras est connue a subi toute la séquence des manipulations conduisant de l'extraction jusqu'à l'obtention des esters butyliques. Dans nos conditions expérimentales, la sous-estimation n'est significative que pour les acides gras de longueur de chaîne inférieure à C12.

Etude de la distribution stéréochimique des acides gras dans les triglycérides. - A partir des extraits lipidiques totaux, les triglycérides sont purifiés par chromatographie préparative sur couche épaisse de gel de silice. Le solvant de migration est un mélange hexane-éther éthylique-acide formique $(80: 40: 1 ; \mathrm{v} / \mathrm{v})$ donnant un Rf de 0,70 pour les triglycérides.

L'étude de la distribution stéréochimique des acides gras dans les triglycérides corporels est réalisée par voie enzymatique. La méthode employée peut être globalement assimilée à celle décrite par Breckenridge (1978). Nous en rappelons brièvement les différentes étapes :

- Une première fraction aliquote de l'échantillon de triglycérides est destinée à la détermination globale de sa composition centésimale en acides gras (pourcentage molaire).

- Une seconde fraction aliquote de l'échantillon est soumise à l'action de la lipase pancréatique de Porc (EC 3.1.1.3.) qui hydrolyse spécifiquement les liaisons esters sn-1 et sn-3. Les 2-monoglycérides résultant sont séparés par chromatographie préparative sur gel de silice. La composition en acides gras de cette fraction est ensuite déterminée par chromatographie en phase gazeuse de façon à obtenir la composition du mélange d'acides gras estérifiés en pósition sn-2.

- Une troisième fraction aliquote de l'échantillon est soumise à l'action d'un réactif de Grignard (bromure d'éthyle-magnésium), de façon à assurer une déacylation " au hasard ". Les $\alpha$ - $\beta$ diglycérides sont séparés des autres composés par 
chromatographie préparative sur gel de silice. A partir des $\alpha-\beta$ diglycérides et de dichlorophosphophénol, on synthétise des $\alpha-\beta$ phosphatidylphénols. Ces derniers sont ensuite soumis à l'action de la phospholipase $A_{2}$ (EC.3.1.1.4; venin de Ophiophagus Hannah Sigma, St-Louis, Etats-Unis) qui hydrolyse spécifiquement la liaison ester en position sn-2 dans les 1,2-diacyl-sn-phosphatidylphénols. Les lysophosphatidylphénols (1-monoacyl-sn-phosphatidylphénols) résultant de la réaction sont séparés des autres composés par chromatographie sur gel de silice additionnée de 5-6\% d'acide borique. L'analyse par chromatographie en phase gazeuse des acides gras permet $d^{\prime}$ obtenir la composition centésimale du mélange estérifié en position sn-1.

La composition centésimale du mélange d'acides gras estérifiés en position sn-3 est ensuite déterminée par le calcul.

Définition du point de fusion des triglycérides. - La méthode employée s'inspire de celle décrite et utilisée par Moore et al. (1969) pour mesurer le point de fusion des lipides totaux des tissus adipeux de Porc. Les triglycérides sont tout d'abord purifiés par chromatographie sur couche épaisse de gel de silice. Les traces de solvant sont éliminées par évaporation prolongée sous vide, puis à environ $40{ }^{\circ} \mathrm{C}$ sous barbottage d'azote. Les échantillons de triglycérides sont introduits dans des tubes capillaires de verre qui sont ensuite scellés, puis solidarisés avec l'extrémité plongeante du corps d'un thermomètre de précision. L'ensemble est plongé dans un bain-marie thermostaté et agité. La température du bain est augmentée degré par degré. L'échantillon est observé à la loupe et sur fond noir et la fusion peut être suivie sur une gamme de plusieurs degrés. Dans le cadre de ce travail, seul le point de fusion totale est considéré, c'est-à-dire la température pour laquelle aucune structure cristalline apparente n'est plus observée.

Etude des phospholipides hépatiques. - Afin de mesurer les éventuels risques d'apparition de carences en acides gras essentiels, la composition en acides gras de la principale classe de phospholipides hépatiques, les phosphatidylcholines, est étudiée. Ces phosphoglycérides sont rassemblés en fonction des différentes conditions nutritionnelles étudiées et des différents âges d'abattage.

La préparation des phosphatidylcholines se fait par chromatographie sur couche mince. Une première chromatographie permet, en utilisant le mélange hexane-éther éthylique-acide formique $(120: 60: 1,5 ; \mathrm{v} / \mathrm{v})$ de séparer l'ensemble des phospholipides des différentes classes de lipides neutres. Une seconde chromatographie, utilisant cette fois le mélange chloroforme-méthanol-acide acétiqueeau $(25: 15: 4: 2 ; \mathrm{v} / \mathrm{v})$ permet de séparer, à partir des phospholipides purifiés précédemment, les phosphatidylcholines des autres classes de glycérophospholipides. Les acides gras des phosphatidylcholines sont ensuite séparés, puis méthylés et analysés par chromatographie en phase gazeuse.

\section{Résultats et discussion.}

1. Correction de la composition en acides gras des lipides totaux corporels.

-- Chez les animaux recevant en permanence le régime de référence $A$, conte- 

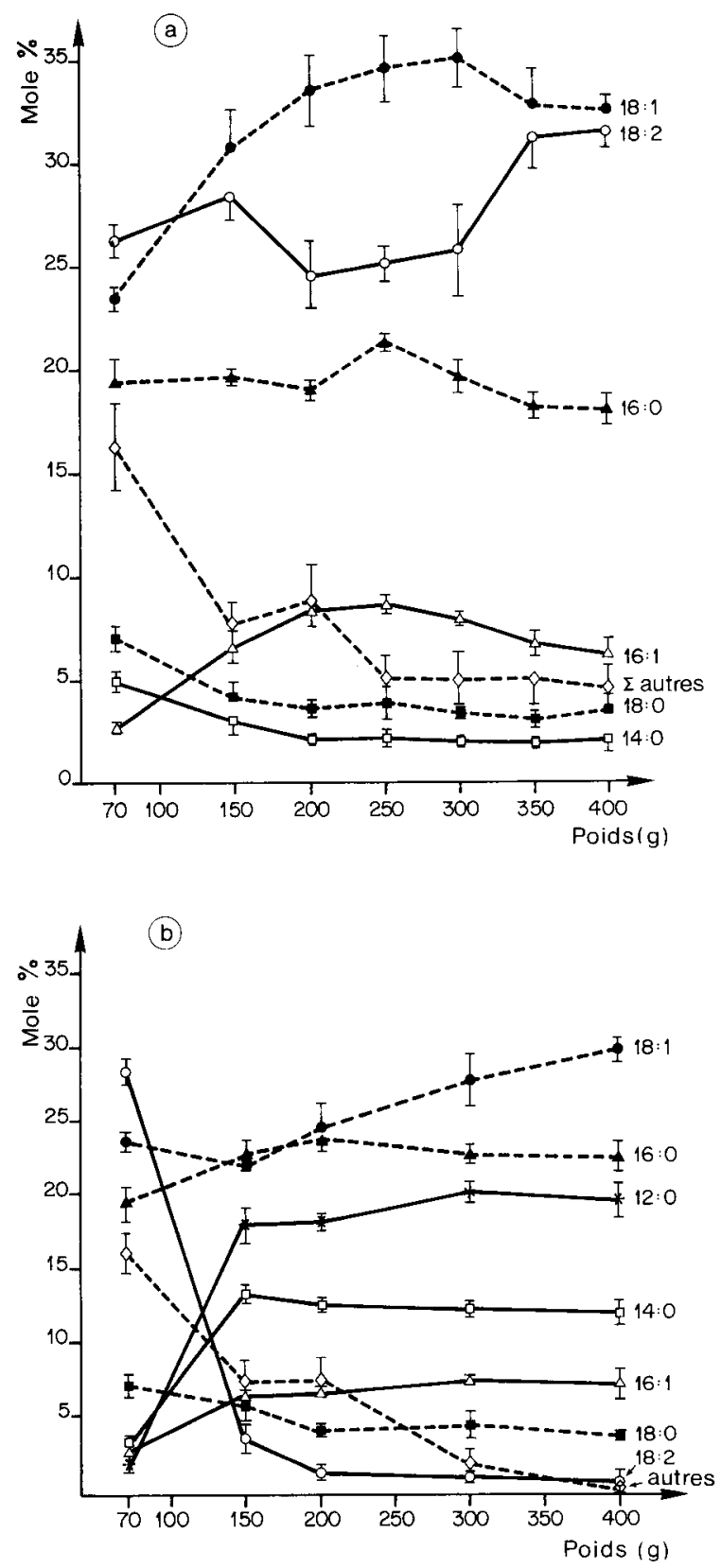

FIG. 1. - Evolution des compositions en acides gras des lipides totaux corpore/s en fonction du poids vif. (a) Animaux recevant le régime $A$ à $4 \%$ d'huile de tournesol. (b) Animaux recevant le régime $B$ à $15 \%$ d'huile de coprah hydrogénée. $\left({ }^{*}\right)$ Acides gras mineurs : chaînes moyennes autres que 12:0, acides gras impairs et ramifiés, acides gras polyinsaturés à chaîne longue $(\geqslant \mathrm{C} 20)$. 
nant $4 \%$ d'huile de tournesol, on remarque tout particulièrement l'accroissement des concentrations en acides oléique (18:1) et linoléique (18:2) entre 70 et $400 \mathrm{~g}$ de poids vif (fig. 1a). Cependant, l'augmentation de la concentration en acide linoléique n'intervient considérablement qu'au-delà de $300 \mathrm{~g}$. Antérieurement, alors que la concentration en acide oléique augmente très sensiblement, celle en acide linoléique se maintient aux environs de $25 \%$ des acides gras totaux (AGT). Ces deux acides gras, qui constituent $50 \%$ des AGT à $70 \mathrm{~g}$, représentent entre 60 et $65 \%$ des AGT à $400 \mathrm{~g}$. Pendant la même période, les concentrations en acides gras saturés, et en particulier celle en acide palmitique (16:0), ne sont que faiblement altérées, tandis que la concentration en acide palmitoléique (16:1) s'accroît légèrement. L'augmentation des concentrations en acides gras insaturés en C18 se fait donc non pas aux dépens des acides gras saturés à chaîne longue $(\geqslant \mathrm{C} 16)$, mais surtout aux dépens des acides gras à chaîne moyenne $(<\mathrm{C} 12)$ et des acides gras mineurs (autres sur la figure 1a).

Chez les animaux recevant le régime de correction $B$ et contenant $15 \%$ d'huile de coprah hydrogénée, on observe, tout d'abord, une diminution extrêmement sévère de la concentration en acide linoléique qui passe d'environ $25 \%$ à $70 \mathrm{~g}$ à moins de $5 \%$ à $150 \mathrm{~g}$ (fig. $1 \mathrm{~b}$ ). Pendant la même période, on note un accroissement très important des concentrations en acide laurique (12:0) et myristique (14:0). La concentration en acide oléique se maintient jusqu'à $150 \mathrm{~g}$, puis augmente légèrement entre 150 et $400 \mathrm{~g}$ de poids vif. Lorsque les animaux atteignent ce poids, les acides gras insaturés en C18 ne représentent plus que $30 \%$ des AGT. Pour sa part, l'acide linoléique ne représente plus qu'environ $1 \%$ des AGT. Enfin, comme cela a déjà été signalé avec le régime $A$, on observe une diminution progressive des concentrations concernant l'ensemble des acides gras mineurs (autres sur la figure $1 b$ ).

Les deux régimes alimentaires testés influencent donc très nettement les caractéristiques des lipides de réserve synthétisés au cours de la croissance. L'emploi du régime $A$ conduit à la synthèse de réserves corporelles riches en acides gras à bas point de fusion (18:1 et 18:2). L'emploi du régime $B$ conduit à l'édification de réserves corporelles riches en acides gras saturés à point de fusion élevé et permet de mettre en évidence l'effet maximal qui peut être attendu de ce type de régime sur les caractéristiques des lipides corporels. En comparant les effets des deux régimes sur les compositions en acides gras à $400 \mathrm{~g}$, on remarque que, par rapport à ce qui est observé avec le régime $A$, le régime $B$ ne modifie que très modérément les concentrations en acide oléique, mais qu'il fait disparaître presque totalement l'acide linoléique. Enfin, il est utile de souligner la rapidité avec laquelle le régime $\mathrm{B}$ modifie la composition en acides gras des lipides corporels.

Lorsque la substitution du régime $B$ au régime $A$ est plus tardive, elle entraîne toujours une baisse des concentrations en acides gras insaturés en $\mathrm{C} 18$, compensée par un accroissement des concentrations en acide laurique (12:0) et myristique (14:0) (fig. 2). Les effets enregistrés au niveau de l'acide oléique d'une part, et de l'acide linoléique d'autre part, sont nettement différenciés. A tous les stades considérés, la substitution a pour effet final de diminuer rapidement et considérablement la concentration en acide linoléique. L'amplitude de cette dimi- 

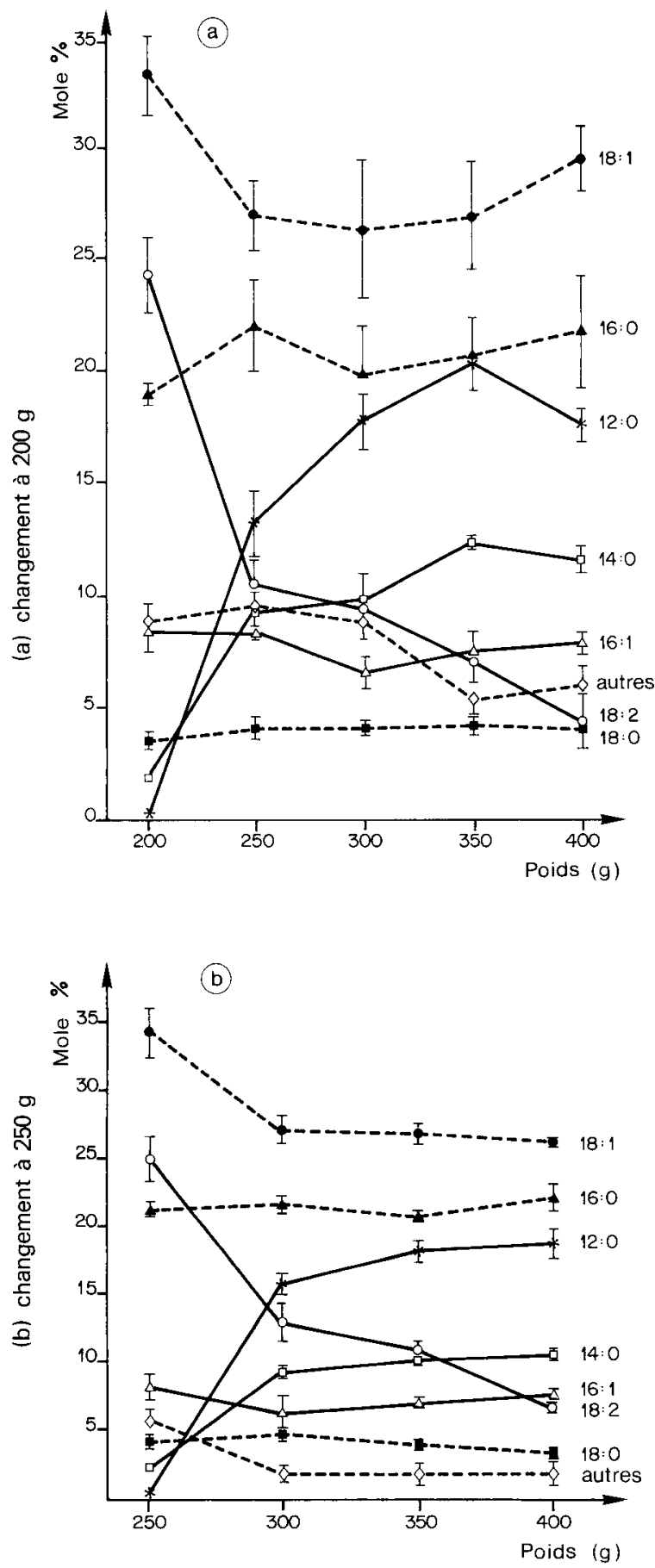

FIG. 2. - (voir légende au verso). 

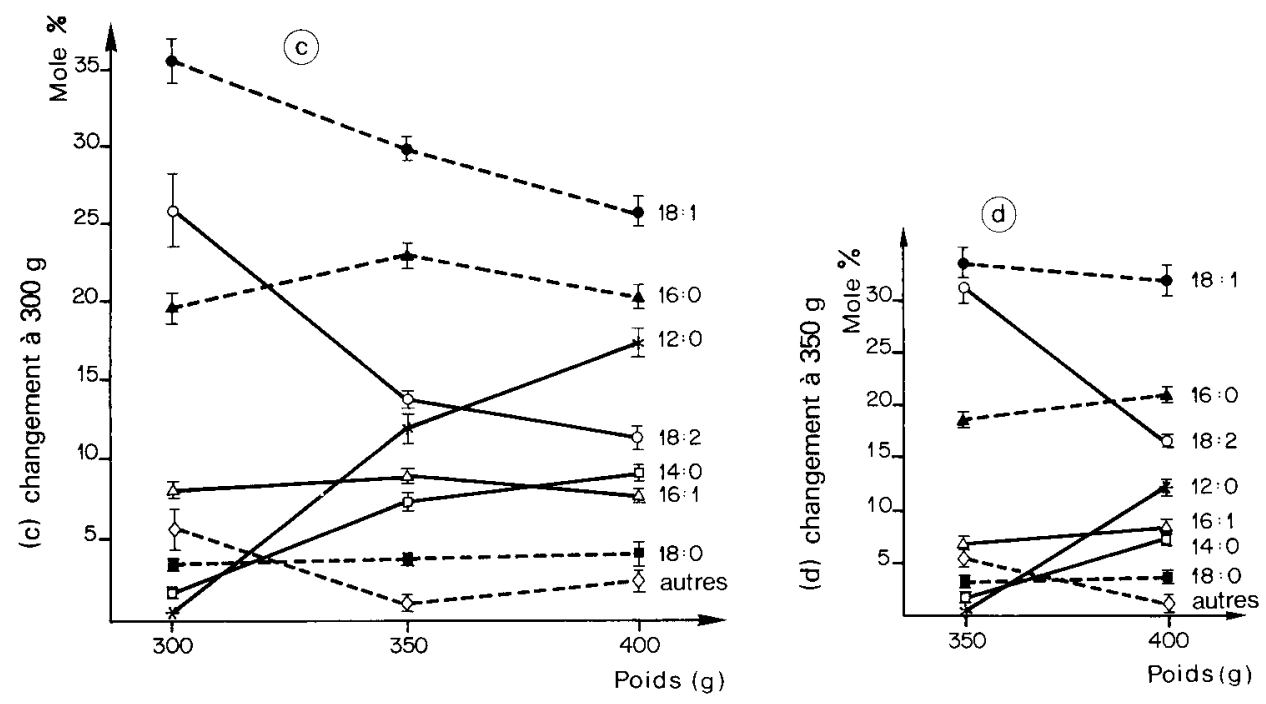

FIG. 2. - Evolution des compositions en acides gras des lipides totaux corporels en fonction du poids vif : effets de la substitution du régime $A$ par le régime $B$ à $200 \mathrm{~g} \mathrm{(a),} 250 \mathrm{~g}(\mathrm{~b}), 300 \mathrm{~g}$ (c) et $350 \mathrm{~g}(\mathrm{~d}) .\left(^{*}\right)$ Acides gras mineurs : chaînes moyennes autres que 12:0, acides gras impairs et ramifiés, acides gras polyinsaturés à chaîne longue $(\geqslant \mathrm{C} 20)$.

nution est d'autant plus forte qu'elle intervient plus précocement. En revanche, la diminution de la concentration en acide oléique entre le début de la substitution et l'abattage à $400 \mathrm{~g}$ est maximum lorsque la substitution a lieu à 250 ou $300 \mathrm{~g}$ (- $23 \%$ relatif), alors qu'aucun effet n'est enregistré lorsqu'elle intervient à $350 \mathrm{~g}$. Par rapport à ce qui est observé à $400 \mathrm{~g}$ lorsque le régime A est utilisé pendant toute la croissance (fig. 1a), la variation des concentrations en acide oléique induite par les différentes substitutions est toujours de faible amplitude, voire même nulle (substitution à $350 \mathrm{~g}$ ). En revanche, les effets sur les concentrations en acide linoléique sont toujours très marqués. Ainsi, même la substitution la plus tardive $(350 \mathrm{~g})$ entraîne une diminution de $50 \%$ de la concentration finale en acide linoléique.

Le tableau 2 permet de comparer les effets des différentes substitutions sur la concentration finale en acides gras insaturés en C18 à $400 \mathrm{~g}$. Par rapport à ce qui est obtenu lorsque les animaux ingèrent le régime $A$ depuis le poids de $70 \mathrm{~g}$, les substitutions intervenant à 200 et $250 \mathrm{~g}$ donnent des résultats similaires et permettent d'induire une diminution proche de $50 \%$ des concentrations en acides gras insaturés en $\mathrm{C} 18$. Si on utilise comme référence l'effet maximum induit à ce niveau par l'ingestion du régime $B$ depuis le poids de $70 \mathrm{~g}$, les substitutions intervenant à 200 et $250 \mathrm{~g}$ permettent d'obtenir environ $90 \%$ de l'effet maximum. Les substitutions qui interviennent à 300 et $350 \mathrm{~g}$ permettent de reproduire respectivement environ 70 et $50 \%$ de l'effet maximum. 80 à $95 \%$ des diminutions des concentrations en acides gras insaturés en C18 engendrées par les diverses substitutions testées s'expliquent uniquement par la décroissance de la concentration en acide linoléique. 


\section{TABLEAU 2}

Comparaison des effets des différentes substitutions sur les concentrations finales en acides gras insaturés en $C 18(400 \mathrm{~g}$ de poids vif).

\begin{tabular}{crrr}
\hline Acides gras & $18: 1$ & $18: 2$ & $\Sigma 18$ ins. \\
\hline Référence A (\% des AGT) $400 \mathrm{~g}$ & 32,5 & 31,5 & 64 \\
Référence B (\% des AGT) $400 \mathrm{~g}$ & 28,5 & 0,5 & 29 \\
\hline $\begin{array}{c}\text { Effet maximum de B par rapport à A } \\
\text { (B - A) }\end{array}$ & $-4,0$ & $-31,0$ & -35 \\
B - A $\times 100$ & $-12,3$ & $-98,4$ & $-54,7$ \\
\hline
\end{tabular}

Effet de $\mathrm{B}_{(200)}{ }^{*}$ par rapport à $\mathrm{A}$

$$
\frac{\left(B_{(200)}-A\right)}{\left(B_{(200)}-A\right)}=100
$$$$
\begin{array}{lll}
-3,5 & -27,5-31
\end{array}
$$$$
-10,8-87,3 \quad-48,4
$$

Effet de $B_{200}$ par rapport à l'effet maximum de B $(\%)$

87,5

88,7

88,6

Effet de $B_{(250)}{ }^{*}$ par rapport à $A$

$$
\begin{aligned}
& \left(B_{(250)}-A\right) \\
& \frac{\left(B_{(250)}-A\right)}{A} \times 100 \\
& \text { A }
\end{aligned}
$$

$-\quad 5,5$

$-25,5$

$-31$

$-16,9$

$-80,9$

$-48,4$

Effet de $B_{250}$ par rapport à l'effet maximum de $B(\%)$

140,0

82,3

88,6

Effet de $B_{(300)}{ }^{*}$ par rapport à $A$

$$
\begin{aligned}
& \left(B_{(300)}-A\right) \\
& \frac{\left(B_{(300)}-A\right)}{A} \times 100
\end{aligned}
$$

$-5,5 \quad-19,5 \quad-25$

Effet de $B_{300}$ par rapport à l'effet maximum de $B(\%)$

$\begin{array}{lll}-16,9 & -61,9 \quad-39\end{array}$

Effet de $B_{(350)}{ }^{*}$ par rapport à $A$

$$
\begin{aligned}
& \left(B_{(350)}-A\right) \\
& \left(B_{(350)}-A\right)
\end{aligned} \times 100
$$

A

Effet de $B_{350}$ par rapport à l'effet maximum de $B(\%)$

140,0

62,9

71,4

${ }^{*} B_{(200)}, B_{(250)} \ldots$ : substitution du régime $B$ au régime $A$, à $200,250,300$ et $350 \mathrm{~g}$.

2. Modifications de la distribution stéréospécifique des acides gras dans les triglycérides de réserve. - La composition en acides gras des triglycérides corporels purifiés et le mode de distribution des acides gras dans 4 conditions différentes sont rapportés (tabl. 3). On a choisi de présenter le cas des animaux de $400 \mathrm{~g}$ ayant ingéré le régime $A$ ou le régime $B$ depuis le poids de $70 \mathrm{~g}$, ainsi que le cas d'animaux de 300 et $400 \mathrm{~g}$ ayant reçu le régime de correction $\mathrm{B}$ à partir de $200 \mathrm{~g}$ (animaux prélevés dans le lot $3.1 \mathrm{du}$ protocole). Pour cette étude, ne sont pris en compte que les acides gras majeurs.

Chez les animaux du groupe 1, les triglycérides se caractérisent par leur teneur élevée en acides gras insaturés en C18 (67\% des AGT). L'acide linoléique (18:2) est estérifié très préférentiellement en position sn-2 et occupe un nombre 
TABLEAU 3

Composition en acides gras des triglycérides purifiés (TG) et distribution stéréospécifique des acides gras dans quatre conditions expérimentales représentatives (\% des acides gras totaux dosés) *

\begin{tabular}{|c|c|c|c|c|c|c|c|c|}
\hline Traitement & & $12: 0$ & $14: 0$ & $16: 0$ & $16: 1$ & 18:0 & 18:1 & $18: 2$ \\
\hline \multirow{4}{*}{$\begin{array}{l}\text { Groupe } 1 \text {, } \\
400 \mathrm{~g}-\text { Régime } \mathrm{A}_{\text {, }} \\
\text { depuis le poids de } 70 \mathrm{~g}\end{array}$} & $\mathrm{TG}$ & - & - & 21,6 & 8,4 & 3,0 & 39,8 & 27,2 \\
\hline & sn-1 & - & - & 32,6 & 11,7 & 11,9 & 28,4 & 15,4 \\
\hline & sn-2 & - & - & 7,7 & 5,7 & 0,3 & 36,6 & 49,7 \\
\hline & sn-3 & - & - & 25,4 & 7,1 & $-3,2$ & 54,4 & 16,5 \\
\hline \multirow{4}{*}{$\begin{array}{l}\text { Groupe } 2 \text {, } \\
400 \mathrm{~g}-\text { Régime } \mathrm{B}, \\
\text { depuis le poids de } 70 \mathrm{~g}\end{array}$} & $\mathrm{TG}$ & 22,1 & 14,2 & 25,3 & 8,1 & 3,5 & 25,5 & 1,3 \\
\hline & sn-1 & 20,9 & 16,2 & 37,3 & 5,6 & 5,6 & 13,5 & 0,9 \\
\hline & $\mathrm{sn}-2$ & 7,5 & 13,3 & 17,0 & 12,1 & 2,0 & 45,7 & 2,4 \\
\hline & sn-3 & 37,9 & 13,1 & 21,6 & 6,6 & 2,9 & 17,3 & 0,6 \\
\hline \multirow{4}{*}{$\begin{array}{l}\text { Groupe } 3-1, \\
300 \mathrm{~g} \text { - Régime } \mathrm{A}, \\
\text { de } 70 \mathrm{~g} \text { à } 200 \mathrm{~g}, \\
\text { puis régime } \mathrm{B}\end{array}$} & TG & 16,3 & 9,9 & 22,4 & 6,1 & 4,5 & 27,8 & 13,0 \\
\hline & sn-1 & 18,0 & 13,3 & 40,7 & 6,8 & 3,8 & 13,5 & 3,9 \\
\hline & sn-2 & 8,8 & 10,9 & 14,5 & 9,2 & 2,1 & 33,4 & 21,1 \\
\hline & sn-3 & 22,1 & 5,5 & 12,0 & 2,3 & 7,6 & 36,5 & 14,0 \\
\hline \multirow{4}{*}{$\begin{array}{l}\text { Groupe } 3-1, \\
400 \mathrm{~g} \text { - Régime } \mathrm{A}, \\
\text { de } 70 \mathrm{~g} \text { à } 200 \mathrm{~g}, \\
\text { puis régime } \mathrm{B}\end{array}$} & TG & 16,8 & 12,7 & 25,3 & 8,6 & 3,5 & 29,4 & 3,7 \\
\hline & sn-1 & 12,8 & 13,7 & 36,5 & 6,9 & 6,2 & 20,8 & 3,1 \\
\hline & sn-2 & 6,1 & 10,4 & 14,6 & 11,2 & 1,3 & 47,6 & 8,8 \\
\hline & $s n-3$ & 31,5 & 14,0 & 24,8 & 7,7 & 3,0 & 19,8 & $-0,8$ \\
\hline
\end{tabular}

* Dans les triglycérides ou sur chaque position.

identique de positions sn-1 et sn-3. L'acide oléique (18:1) est distribué sur les 3 positions, mais avec une préférence pour la position sn-3. L'acide palmitique (16:0) est préférentiellement estérifié sur les positions sn-1 et sn-3. Seulement $12 \%$ de l'acide palmitique total estérifié sont retrouvés en position sn-2. L'acide palmitoléique (16:1) est préférentiellement estérifié en position sn-1 et, enfin, l'acide stéarique (18:0) est exclusivement estérifié en position sn-1.

Chez les animaux du groupe 2, soumis pendant toute la durée de leur croissance au régime $\mathrm{B}$, de profondes modifications du mode de distribution des acides gras sont observées par rapport au groupe 1 . Plus de $88 \%$ de l'acide laurique (12:0) estérifié se retrouvent sur les positions sn-1 et sn-3 et plus de $57 \%$ uniquement en position sn-3. L'acide myristique (14:0) est distribué d'une façon similaire sur les 3 positions. L'acide palmitique (16:0) maintient son pourcentage d'estérification en position sn-1 (49\% dans les 2 cas) mais, en revanche, la diminue pour la position sn-3 ( $28 \%$ contre 39$)$ et l'augmente pour la 
position sn-2 (22\% contre 12). L'acide palmitoléique (16:1) est très préférentiellement estérifié en position sn-2 et symétriquement distribué entre les positions sn-1 et sn-3. L'acide stéarique (18:0) est toujours préférentiellement estérifié sur les positions sn-1, mais occupe également les positions sn-2 et sn-3 (près de $50 \%$ de cet acide se retrouve sur ces deux positions). L'acide oléique (18:1) est très préférentiellement estérifié en position sn-2 $(60 \%)$, le reste étant réparti d'une façon presque symétrique entre les positions sn- 1 et sn-3. Enfin, l'acide linoléique (18:2), bien que présent à une très faible concentration, conserve son mode d'estérification préférentielle sur la position sn-2. II est donc clair que l'ingestion précoce et prolongée d'huile de coprah entraîne des modifications sensibles du mode traditionnel de distribution des acides gras au sein des triglycérides de réserve tel qu'il a pu être décrit antérieurement (Brockerhoff, Hoyle et Wolmark, 1966). Ces modifications découlent essentiellement des variations des concentrations respectives de chaque acide gras et de l'estérification de quantités importantes d'acides gras normalement absents des dépôts adipeux du rat adulte ou présents à des concentrations très faibles $(12: 0$ et $14: 0$, en particulier). Enfin, elles dépendent du mode d'estérification de l'acide laurique qui est très préférentiel vis-à-vis des positions sn-1 et sn-3, et ne peut, en aucune façon, être assimilé à une distribution " au hasard ").

Pour les animaux du groupe 3.1 abattus à $400 \mathrm{~g}$, le mode de distribution des acides gras peut être assimilé à celui qui est observé chez les animaux du groupe 2. Chez les animaux du groupe 3.1 abattus au poids vif de $300 \mathrm{~g}$, on observe un mode de distribution qui pourrait être le reflet de l'existence de 2 groupes de triglycérides dont l'un correspondrait à un ensemble résiduel de molécules synthétisées avant $200 \mathrm{~g}$ (ingestion du régime A). D'après les concentrations en chaque acide gras dans les triglycérides provenant de ce lot, ces deux groupes correspondraient respectivement à environ $35 \%$ et $65 \%$ de triglycérides de type "régime $A$ » et "régime $B$ ». En revanche, le calcul ne permet pas de retrouver un mode de distribution intramoléculaire compatible, surtout pour les acides palmitique (16:0), oléique (18:1) et linoléique (18:2). Dans le cadre de cette hypothèse, on pourrait envisager que des redistributions, tout au moins partielles, des acides gras primitivement estérifiés avant $200 \mathrm{~g}$ de poids vif, aient pu intervenir entre 200 et $300 \mathrm{~g}$ de poids vif.

3. Effets sur les températures apparentes de fusion totale des triglycérides de réserve. - Au cours de la croissance, entre 200 et $400 \mathrm{~g}$ de poids vif, et lorsque les animaux ingèrent le régime $A$, on observe parallèlement à l'enrichissement des lipides corporels en acide linoléique une modeste diminution de la température apparente de fusion totale (fig. 3). Cependant, durant cette période, les teneurs totales en acides gras insaturés ne sont pas significativement modifiées.

La substitution du régime $B$ au régime $A$, quel que soit le poids auquel elle intervient, modifie simultanément les teneurs en acides gras mono et polyinsaturés et les températures de fusion des triglycérides de réserve. Par rapport à ce qui est observé chez les animaux témoins ayant ingéré le régime $\mathrm{A}$ pendant toute la croissance, les substitutions les plus précoces (à 200 et $250 \mathrm{~g}$ ) 


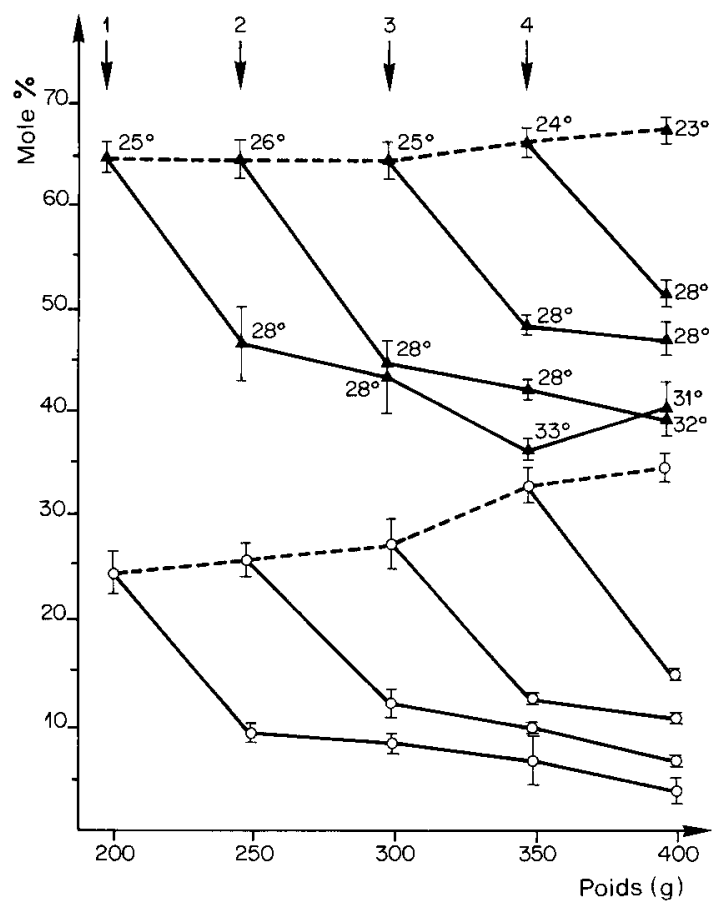

FIG. 3. - Evolution des concentrations en acides gras insaturés ( $\mathbf{\Delta}$ ) en C18 et en acide linoléique (U) en fonction du poids vif des animaux lors de la substitution. Effets sur la température apparente de fusion des triglycérides corporels correspondants.

augmentent de 8 à $9{ }^{\circ} \mathrm{C}$ le point de fusion totale apparent des triglycérides à $400 \mathrm{~g}$, alors que les plus tardives (à 300 et $350 \mathrm{~g}$ ) l'augmentent encore d'environ $5{ }^{\circ} \mathrm{C}$. II semblerait donc que par intervention nutritionnelle sur une période de croissance représentant le huitième de la croissance totale de l'animal, il serait possible d'augmenter d'au moins $5^{\circ} \mathrm{C}$ le point de fusion des triglycérides de réserve.

4. Conséquences des différents traitements nutritionnels sur les phospholipides hépatiques. Recherches d'éventuelles carences en acides gras essentiels. Chez les animaux recevant le régime $A$, les concentrations en acides linoléique et arachidonique se maintiennent tout au long de la croissance (tabl. 4.1); de plus, la teneur en $\mathrm{C} 20: 3 \mathrm{n}-9$ demeure inférieure à $0,1 \%$. Quand les animaux ingèrent le régime $B$ à partir du poids vif de $70 \mathrm{~g}$, on observe très rapidement une chute des concentrations en acides linoléique et arachidonique et une élévation symétrique des concentrations en acide éicosatriénoïque (20:3 n-9). Le rapport triène/tétraène de 0,4 , indiquant que les animaux sont carencés en acides gras essentiels (Holman, 1968), est atteint dès $150 \mathrm{~g}$ et évolue ensuite entre 0,75 et 1,02 . 


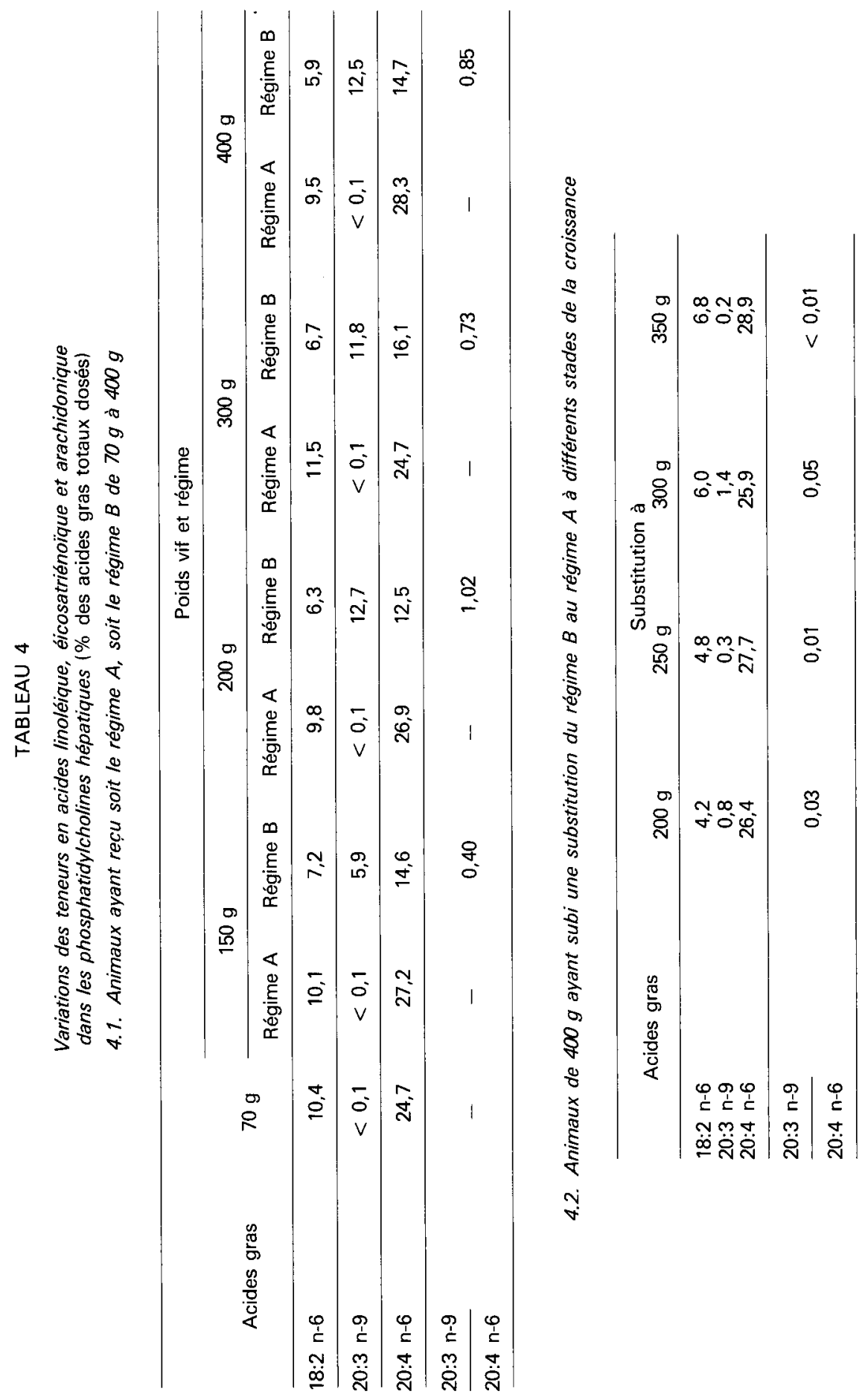


Lorsque la distribution du régime B commence plus tardivement (tabl. 4.2), la concentration finale en acide linoléique est toujours plus faible que celle observée chez les animaux ayant continué à ingérer le régime $A$ pendant leur croissance. En revanche, les teneurs en acide arachidonique sont voisines. La présence d'acide éicosatriénoïque est toujours mise en évidence, mais le support triène/tétraène n'atteint jamais la valeur de 0,4 qui permettrait de suspecter l'existence d'une carence en acides gras essentiels.

Le régime de correction $B$ n'induit donc pas de carence en acides gras essentiels, lorsqu'il est fourni à des animaux d'au moins $200 \mathrm{~g}$ de poids vif et qui ont reçu antérieurement un régime permettant de couvrir largement leurs besoins.

\section{Conclusion.}

L'emploi d'un régime riche en coprah hydrogéné (environ $50 \%$ d'acide laurique) permet donc de diminuer considérablement et très efficacement les teneurs en acide linoléique des lipides de réserve du rat en croissance. Son utilisation, même tardive (dernier huitième de la croissance totale), conduit à des résultats très intéressants dans ce domaine et qui se traduisent par des améliorations significatives des propriétés physiques des graisses produites. L'acide laurique s'estérifie préférentiellement sur les positions " externes " et surtout en position sn-3 dans les triglycérides de réserve. En cela, son mode d'estérification peut être jugé comme voisin de celui qui est observé au niveau des triglycérides du lait de rongeur pour l'acide laurique endogène synthétisé " in situ » dans la glande mammaire (Tanioka et al., 1974 ; Lin et al., 1976). L'estérification d'acide laurique dans les triglycérides corporels entraîne des modifications sensibles du mode de distribution stéréospécifique d'autres acides gras. Ces modifications de composition d'une part, et de distribution intramoléculaire d'autre part, se traduisent par une augmentation des points de fusion des triglycérides par rapport à ce qui est observé chez les témoins.

Chez le Rat, l'utilisation de rations riches en coprah hydrogéné n'entraîne pas l'apparition de carence en acides gras essentiels en $\mathbf{n}-6$ dans la mesure où les animaux reçoivent ces régimes après $200 \mathrm{~g}$, et qu'ils ont reçu antérieurement un régime équilibré. Dans une publication ultérieure nous présenterons les résultats d'un travail du même type entrepris cette fois chez le Porc.

Reçu en novembre 1983. Accepté en février 1984.

\section{Références}

BRECKENBRIDGE W. C., 1978. Stereospecific analysis of triacylglycerols, 197-232. In KUKSIS A., Handbook of lipid research, Vol. 1, Fatty acids and glycerides, Plenum Press, New York and London.

BROCKERHOFF H., HOYLE J. H., WOLMARK N., 1966. Positional distribution of fatty acids in triglycerides of animal depot fats. Biochim. biophys. Acta, 116, 67-72. 
CHRISTIE W. W., MOORE J. H., 1970. A comparaison of the structures of triglycerides from various pig tissues. Biochim. biophys. Acta, 210, 46-56.

DEMARNE Y., TOURE M., DESNOYERS F., VODOVAR N., FLANZY J., 1974 . Evolution des réserves lipidiques du jeune rat en croissance en fonction du temps et de la qualité des lipides ingérés. Ann. Biol. anim. Bioch. Biophys., 14, 793-811.

DEMARNE Y., TOURE M., FLANZY J., LECOURTIER M. J., 1975. Influence du degré d'insaturation des lipides alimentaires sur la croissance et la lipogenèse chez le Rat. Nutr. Métab., 19, 28-48.

DEMARNE Y., PERAZA-CASTRO C. E., HENRY Y., FLANZY J., 1977. Effets de l'ingestion de tridodécanoate de glycérol (Tri C12:0) sur la composition en acides gras des lipides de différents tissus adipeux chez le Porc. Ann. Biol. anim. Bioch. Biophys., 17, 137-146.

DEMARNE Y., EPO N., FLANZY J., LECOURTIER M. J., 1978. Comparison of long-term lipogenic effects of two different medium chain triglycerides (Tri C18:0 and Tri $\mathrm{C} 12: 0$ ) in the growing rat. Arch. int. Physiol. Bioch., 86, 25-35.

DEMARNE Y., THOUARD D., PIHET A., LECOURTIER M. J., 1981. Effect of different levels of dietary fats upon the positional distribution of fatty acids in rat body triacylglycerols. Comp. Biochem. Physiol., 68 (A), 361-371.

FLANZY J., FRANÇOIS A. C., RÉRAT A., 1970, Utilisation des acides gras chez le Porc. Ann. Biol. anim. Bioch. Biophys., 10, 603-620.

FLANZY J., RÉRAT A., FRANC̣OIS A. C., 1968. Etude de l'utilisation digestive des acides gras chez le Porc. Ann. Biol. anim. Biophys., 8, 537-540.

FOLCH J., LEES M., SLOANE-STANLEY G. H., 1957. A simpie method for the isolation and purification of total lipids from tissues. J. biol. Chem., 226, 497-509.

HOLMAN R. T., 1968. The ratio of trienoic-tetraenoic acids in tissue lipids as a measure of essential fatty acid requirement. $J$. Nutr., 70, 406-287.'

LIN C. Y., SMITH S., ABRAHAM S., 1976. Acyl specificity in triglycerides synthesis by lactating rat, mammary gland. J. Lipid Res., 17, 647-656.

MOORE J. H., CHRISTIE W. W., BRAUDE R., MITCHELL K. G., 1969. The effect of dietary copper on the fatty acid composition and physical properties of pig adipose tissues. Br. J. Nutr., 23 , 281-287.

PARISOT A., 1949. In Constantes et données numériques des corps purs de la chimie des corps gras. DUNOD Ed., Paris.

SELLIER P., 1983. Effets de la sélection sur l'adiposité chez le Porc. Rev. franç. Corps Gras, 30 , $103-111$.

TANIOKA H., LIN L. Y., SMITH S., ABRAHAM J., 1974. Acyl specificity in glyceride synthesis by lactating rat mammary gland. Lipids, 9, 229-234. 